\title{
An evaluation of mini-laparotomy cholecystectomy in the laparoscopic era: a rural experience
}

\author{
A Balasubramanian, ${ }^{1}$ S Cheddie, ${ }^{1}$ N M Naidoo, ${ }^{2}$ B Singh ${ }^{1}$ \\ ${ }^{1}$ Department of Surgery, University of KwaZulu-Natal \\ ${ }^{2}$ Department of General Surgery, University of KwaZulu-Natal
}

Corresponding author: Shalen Cheddie (scheddie@gmail.com)

\begin{abstract}
Background: Laparoscopic cholecystectomy (LC) is the gold standard for the management of symptomatic cholelithiasis and complications of gallstone disease. Mini laparotomy cholecystectomy (MOC) may be a more appropriate option in the resource constrained rural setting due to its widespread applicability and comparable outcome with LC. The study aimed to provide an epidemiological analysis of gallstone disease in the rural population and to evaluate the outcome of MOC in a rural hospital.

Methods: A retrospective chart analysis of 248 patients undergoing cholecystectomy in a rural regional referral hospital in KwaZulu-Natal from January 2009 to December 2013 was undertaken.

Results: Of the 248 patients, the majority were females $(n=211,[85 \%])$. The most frequent indications for cholecystectomy included: biliary colic $(n=115,[46.3 \%])$; acute cholecystitis $(n=80,[32.3 \%])$; gallstone pancreatitis $(n=27,[10.8 \%])$. Forty cases $(16.1 \%)$ were converted to open cholecystectomy (OC). The median operative time was 40 minutes (range18-57). Twenty-three morbidities $(9.3 \%)$ occurred including: bile leaks $(n=6,[2.4 \%])$; bleeding from drain site $(n=1,[0.4 \%])$, incisional hernia $(n=8[3.2 \%])$ and wound sepsis $(n=8[3.2 \%])$. The median length of hospital stay in patients who underwent MOC was 48 hours (range: $24-72$ hours) and the median time to return to work was 10 days (range: 4-14 days). There was one mortality in the entire cohort.

Conclusion: MOC is a safe and feasible operation for symptomatic cholelithiasis when cholecystectomy is indicated. The low operative morbidity and mortality in the context of a high risk patient profile and complicated gallstone disease makes this procedure an alternative to LC where LC is inaccessible.
\end{abstract}

Key words: Mini-cholecystectomy; laparoscopic cholecystectomy; outcome; open cholecystectomy; rural

\section{Introduction}

Advances in technology and instrumentation for laparoscopic surgery has ensured that laparoscopic cholecystectomy (LC) is presently regarded as the "gold standard" treatment for the management of symptomatic cholelithiasis and other complications of gallstone disease. ${ }^{1}$ The main advantages of LC over open cholecystectomy (OC) are reduced analgesic requirements, shorter hospital stay and quicker return to work. ${ }^{2}$ The main drawback of LC in some middle and lower income countries, particularly in the rural setting is the need for appropriate technical expertise, expensive equipment and consumables which limit the widespread use of this procedure.

Prior to the wide usage of LC, a randomised control trial had successfully established the superior efficacy of minicholecystectomy (MOC) over the traditional OC with a shorter hospital stay and less analgesic requirements being reported. ${ }^{3}$ A landmark randomised control trial comparing MOC to LC by Majeed et al. demonstrated that LC took longer to undertake than MOC and did not have any significant advantages in terms of hospital stay or postoperative recovery. ${ }^{4}$ For these reasons, it is suggested that MOC may be the preferred surgical option in a resource constrained rural environment lacking technical expertise and appropriate equipment.

South Africa is considered a middle to lower income country with a substantial rural population. The role of MOC in the management of gallstone disease has not been evaluated in the South African rural context. The aim of this study was to provide an epidemiological analysis of gallstone disease in a South African rural population and to evaluate the outcome of MOC in this setting. 


\section{Methods}

Ethical approval to conduct the study was obtained from the Biomedical Research Ethics Committee of the University of KwaZulu-Natal (BE 077/15). A retrospective chart analysis of 248 adult patients ( $\geq 16$ years) planned for a MOC at a single surgical unit at Madadeni Hospital, a regional referral hospital in rural KwaZulu-Natal, from January 2009 to December 2013 was undertaken. MOC was routinely done due to a lack of laparoscopic equipment during the period of this study.

All patients with symptomatic gallstones underwent a MOC. Patients with acute cholecystitis, irrespective of duration of symptoms were managed by intravenous antibiotics and had a cholecystectomy on the next available operating list, which ranged from 24 hours to 96 hours post admission.

Patients with suspected or proven common bile duct stones were referred to a tertiary centre for further investigation and were returned for elective cholecystectomy once the common bile duct (CBD) was clear of stones.

Patients with mild gallstone pancreatitis underwent a MOC during the same hospital admission.

\section{The MOC technique}

The MOC was performed by a consultant surgeon with one assistant. The surgical technique of the MOC involved an incision that did not exceed $6 \mathrm{~cm}$ in length and was placed over the lateral margin of the rectus muscle $2 \mathrm{~cm}$ below and parallel to the costal margin. Following the incision of the abdominal wall aponeurosis, the abdominal wall musculature was split up to the lateral rectus abdominis border to gain access to the peritoneal cavity. Two Deaver retractors ( $25 \mathrm{~mm}$ width) were strategically placed to facilitate adequate exposure of Calot's triangle. Raytec gauze swabs $(35 \times 10 \mathrm{~cm})$ rather than tape swabs were used to enhance the exposure and dissection of Calot's triangle and haemostasis of the gallbladder bed.

The gallbladder was routinely decompressed via needle aspiration. Gallbladder laden with gallstones or harbouring impacted gallstones in Hartmann's pouch were evacuated via a cholecystostomy. Spillage of gallstones was minimised by the safe placement of Raytec swabs around the gallbladder. A Calot's triangle first approach was used to define the cystic duct and artery; a fundus first approach was used where dense adhesions or impacted stones in Hartmann's pouch precluded safe dissection of Calot's triangle. Cystic artery and duct were consistently ligated. The subhepatic space was routinely drained by a closed tube drainage system. A mass closure technique using loop nylon was used to effect closure of the abdominal incision.

Intramuscular opioids were routinely administered only for the first 24 hours post MOC. Thereafter patients were placed on Diclofenac intramuscularly and oral paracetamol until discharged.

Variables evaluated in the data analysis were the age and gender of the patient and indication for cholecystectomy.

The medical co-morbidities evaluated included: cardiac disease (ischaemic heart disease, congested cardiac failure and hypertension), diabetes mellitus, chronic obstructive airways disease, acute and chronic renal failure, previous stroke and body mass index (BMI). The BMI was considered as obese (BMI 30-40) and morbidly obese (BMI > 40). The operative time and the indication for conversion to traditional OC were also recorded. The outcome measures included the following: length of hospital stay, return to work, 14 day mortality and morbidity.

Bile duct injury, bile leak, wound sepsis and incisional hernia were graded according to the Clavien-Dindo Classification of Surgical complications. ${ }^{5}$

Patients were followed up at 2 weeks for wound review and gallbladder histology. Statistical analysis was performed using GraphPad software (GraphPad, LaJolla, USA). Categorical variables were compared using the Chi-squared test. A p-value of $<0.05$ was considered statistically significant.

\section{Results}

Of the 248 patients who underwent surgery, there were 37 males $(14.9 \%)$ and 211 females $(85 \%)$. The median age was 45 years (range 17-78 years). The associated medical comorbidities are listed in Table 1.

The indications for cholecystectomy are outlined in Table 2. Males $(75.7 \%)$ presented with more severe complications of

\begin{tabular}{lc} 
Table 1: Associated medical co-morbidities in study \\
population (n=248) \\
\hline Medical co-morbidity & n (\%) \\
\hline Cardiac disease* & $22(8.8)$ \\
Diabetes mellitus & $96(38.7)$ \\
Chronic Respiratory Disease & $10(4)$ \\
Renal impairment: Acute & $22(8.8)$ \\
Renal impairment: Chronic & $6(2.4)$ \\
Previous CVA & $5(2)$ \\
Obese & $125(50.4)$ \\
Morbid obesity & $(28.6)$ \\
CVA: cerebrovascular accident & \\
*Include hypertension, congestive cardiac failure, ischaemic heart \\
disease
\end{tabular}

Table 2: Indication for surgery in male and female cohorts $(\mathrm{n}=\mathbf{2 4 8})$

\begin{tabular}{lcc}
\hline Indication for surgery & Female n (\%) & Male n (\%) \\
\hline Biliary Colic & $96(38.6)$ & $9(3.6)$ \\
Acute Cholecystitis & $60(24.1)$ & $20(8.1)$ \\
Gallstone Pancreatitis & $26(10.4)$ & $1(0.4)$ \\
$\begin{array}{l}\text { Choledocholithiasis } \\
\text { (post ERCP) }\end{array}$ & $18(7.2)$ & $2(0.8)$ \\
Acalculous Cholecystitis & $8(3.2)$ & $4(1.6)$ \\
Cholecystoduodenal Fistula & $3(1.2)$ & $1(0.4)$ \\
ERCP: endoscopic retrograde cholangiopancreatography
\end{tabular}




\begin{tabular}{lcc}
$\begin{array}{l}\text { Table 3: Morbidities according to Clavien-Dindo Classification } \\
\text { of Surgical Complications. }\end{array}$ & Grade & n (\%) \\
\hline & 4 & 0 \\
\hline Bile duct injury & 1 & $6(2.4)$ \\
Bile leak & 2 & $7(2.8)$ \\
Wound sepsis & 3 & $1(0.4)$ \\
Severe wound sepsis & 3 & $8(3.2)$ \\
Incisional hernia & &
\end{tabular}

gallstone disease, as compared to females (45.5\%), $\mathrm{p}-<-0.05$.

Forty patients $(16 \%)$ were converted from MOC to OC. The indications for conversion included: bleeding from the liver bed $(n=15)$; dense adhesions in the Calot's triangle $(n=12)$; Mirrizi's syndrome Type $1(\mathrm{n}=10)$; and impacted cystic duct stones $(\mathrm{n}=3)$.

The median operative time was 40 minutes (range 18-56 minutes).

The median length of hospital stay in patients who underwent MOC was 48 hours (range 24-72) and the median time to return to work was 10 days (range 4-14).

Seven Grade 1 morbidities $(2.8 \%)$ occurred in patients who underwent MOC. These included bile leaks $[\mathrm{n}=6(2.4 \%)]$ which resolved spontaneously and bleeding $[\mathrm{n}=1(0.4 \%)]$ from the drain site which stopped spontaneously. Seven Grade 2 morbidities included wound sepsis $3.2 \%$ )]. All patients who developed wound sepsis had presented with acute cholecystitis; risk factors included diabetes mellitus and obesity. These patients were treated with antibiotics and topical povidone iodine dressings. There were no CBD injuries recorded. Nine Grade 3 morbidities included 8 incisional hernias $(2.8 \%)$. Obesity was a risk factor in all these cases. All underwent a tissue repair electively. One patient with severe wound sepsis (Grade 3) required a debridement in theatre under general anaesthesia (Table 3).

One patient died 8 hours postoperatively from a myocardial infarction. The patient had diabetes mellitus without an antecedent history of cardiac disease.

\section{Discussion}

MOC was initially described by Dubois and Berthelot in 1982 and its popularity grew in the 1980s and early 1990s following the recognition that the classic large subcostal incision could be safely and effectively replaced by a much smaller incision with the benefit of a shorter convalescence period. ${ }^{3,6,8,9}$ In our resource constrained practice, $\mathrm{LC}$ is often not feasible and it is largely for this reason we performed MOC as opposed to the classic OC.

In this report, the length of the subcostal incision used was consistently $6 \mathrm{~cm}$; however, in the literature the reported length of skin incision undertaken for MOC ranges from $3-8 \mathrm{~cm} .{ }^{6,7}$
A recent meta-analysis of randomised control trials has shown that LC was associated with a longer operative time and shorter hospital stay than MOC. All other postoperative outcome measures were similar in both groups. ${ }^{10}$ Vagenas et al. have shown that cholecystectomy through a mini-laparotomy incision is a lower-cost, versatile, and safe alternative to LC. ${ }^{11}$ However, there was a shorter length of hospital stay and return to normal activities in the LC group.

A randomised control trial by Barkun et al. have shown that the median length of hospital stay in LC group vs the MOC group was 3 vs 4 days and time to convalescence was 11.9 vs 22 days, respectively. ${ }^{12}$ A randomised trial by Kiviluoto et al. have shown that LC for acute cholecystitis was associated with a 4-day hospital stay vs a 6-day hospital stay with MOC and that the time of sick leave was 13 days with LC vs 30 days with MOC. ${ }^{13}$ Interestingly, Majeed et al. have reported that MOC may be performed as day-care or as ambulatory surgery. ${ }^{4}$

In this report, the median length of hospital stay and the mean time to return to work was very comparable to LC despite the majority of patients having significant medical comorbidity. Forty $(16 \%)$ of MOC were converted to traditional OC (15 cm skin incision). The main reason for conversion was the inability to safely dissect Calot's triangle as a result of dense adhesions, bleeding and stone impaction at the Hartmann's pouch. Headlamps were not available and this may have aided us in visualisation and dissection as has been described by other proponents of MOC. ${ }^{7}$

Shulutko et al. reported a conversion rate of $3.7 \%$ from MOC to OC especially when surgery was done for acute cholecystitis. ${ }^{14}$ Mbatha et al. have shown $44 \%$ of patients who underwent a LC at an urban level 2 hospital in Kwa-Zulu Natal presented with biliary colic with a low conversion rate of $5.4 \% .^{15}$ The majority of patients in our study $(56.2 \%)$ had complicated disease at presentation accounting for the high conversion rate to traditional OC. LC for cholecystitis has been associated with a higher conversion rate of up to $22 \% .{ }^{16}$ In this study, males undergoing MOC had a higher percentage of complicated gallstone disease. Male gender is a recognised risk factor for complicated LC. ${ }^{17,18}$ Amber et al. have suggested that the higher pain threshold in males may account for the delay in presentation and repeated episodes of cholecystitis. ${ }^{19}$ The safe completion of a LC also hinges on this vital principle of accurate delineation of the anatomy of Calot's triangle. LC for complicated gallstone disease is more challenging and often demands superior laparoscopic skills and equipment such as endo-loops and endo-retrieval bags. MOC may be a reasonable alternative to LC particularly in the context of complicated gallstone disease due to its wider applicability and lower demand on surgical equipment.

In this study, the median operative time for MOC was 40 minutes (range 18-56 minutes) which is comparable to other studies by Chalkoo et al., which showed a median operative time of 35 minutes (range 35-110 minutes), and 
Shulutko et al. who have reported a median operative time of $65.4 \mathrm{~min} \pm 24.5 \mathrm{~min} .{ }^{7,14}$ Other studies have shown that the operative time for LC is significantly longer than MOC. .,6,10,20 $^{2}$ It is suggested that in a resource constrained setting, the shorter operative time with MOC may allow for the more efficient utilisation of theatre resources.

CBD injury is regarded as the most devastating complication of cholecystectomy; the incidence of this complication has increased from 0.2 to $0.4 \%$ with the introduction of laparoscopic surgery. ${ }^{21}$ In our series of MOC there was no bile duct injury recorded. The commonest morbidity was bile leaks $(8 / 248)$ that resolved spontaneously without the need for further imaging or endoscopic intervention. The safe management of bile leaks was facilitated by routine use of drains in all patients. There was a low incidence of wound sepsis $(3.2 \%)$ despite the high risk population group with obesity (78\%) and diabetes mellitus (38.7\%), which present major risk factors for wound sepsis. It has been suggested that overzealous traction of the wound edges may predispose to tissue injury with consequent wound infection. ${ }^{7}$

Cost factor analysis is essential in the provision of long term sustainable health care services. MOC seems to be the preferred operative technique over the laparoscopic technique both from a hospital and societal cost perspective. ${ }^{22}$ Most of the societal cost is associated with sick leave taken from employed patients. In the rural public hospital setting, the majority of indigent patients are unemployed, making length of hospital stay and return to work weak outcome measures when comparing to the Western literature. Calvert et al. have also shown that MOC costs $26 \%$ less than LC with cost of equipment and operations themselves accounting for most of the difference. ${ }^{23}$

The limitations of this study include the rural study location, which may have accounted for the more advanced clinical presentation of gallstone disease due to the lack of accessibility to health care facilities. The MOC was performed by a single consultant surgeon at a single centre, thus the findings cannot be generalised to all surgical departments. Surgical outcomes will vary depending on the expertise of the operating surgeon. The employment status of patients undergoing surgery was not recorded, thus convalescence time (with respect to time to return to work) could not be accurately assessed.

\section{Conclusion}

MOC is a safe and feasible operation for symptomatic cholelithiasis when cholecystectomy is indicated. The low operative morbidity and mortality especially in the context of a high risk patient profile and complicated disease presentation makes this procedure an attractive alternative to LC when inaccessible. With the increasing deployment of surgeons to rural facilities, training centres must ensure that surgical trainees attain proficiency in undertaking this useful technique for a common surgical condition.

\section{Conflict of interest}

The authors do not have a conflict of interest to share

\section{REFERENCES}

1. Singh B, Moodley J, Haffejee AA, Naidu AG, Rajaruthnam P. Laparoscopic cholecystectomy--an audit from Durban. S Afr J Surg. 1998;36(1):17-21.

2. Karim T, Kadyal A. A Comparative Study of Laparoscopic vs. Open Cholecystectomy in a Suburban Teaching Hospital. J Gastrointest Dig Syst. 2015 (accessed on: 21 Dec 2016);5(6). https://dx.doi.org/10.4172/2161-069X.1000371

3. O'Dwyer J, McGregor JR, McDermott EWM, Murphy JJ, O'Higgins NJ. Patient recovery following cholecystectomy through a $6 \mathrm{~cm}$ or $15 \mathrm{~cm}$ transverse subcostal incision: a prospective randomized clinical trial. Postgrad Med J. 1992;68(804):817-9. https://doi.org/10.1136/pgmj.68.804.817

4. Majeed AW, Troy G, Nicholl JP, et al. Randomised, prospective, single-blind comparison of laparoscopic versus small-incision cholecystectomy. Lancet 1996;347(9007):989-94. https://doi. org/10.1016/s0140-6736(96)90143-9

5. Dindo D, Dimartinez N, Clavien PA. Classification of Surgical Complications. Ann Surg. 2004;240(4):205-13. https://doi. org/10.1097/01.sla.0000133083.54934.ae

6. Ros A, Gustafsson L, Krook H, et al. Laparoscopic cholecystectomy versus mini-laparotomy cholecystectomy Ann Surg. 2001;234(6):741-9. https://doi.org/10.1097/00000658200112000-00005

7. Chalkoo M, Ahangar S, Duranni AM, Chalkoo S, Shah MJ, Bashir MI. Mini-lap cholecystectomy: modifications and innovations in technique. Int J Surg, 2010;8(2):112-7. https:// doi.org/10.1016/j.ijsu.2009.11.007

8. Assalia A, Schein M, Kopelman D, et al. Mini-cholecystectomy vs conventional cholecystectomy: prospective randomized trial-implications in the laparoscopic era. World J Surg. 1993;17(6):755-9. https://doi.org/10.1007/bf01659087

9. Schmitz R, Rohde V, Treckmann J, et al. Randomized clinical trial of conventional cholecystectomy versus minicholecystectomy. Br J Surg. 1997;84(12):1683-6. https:// doi.org/10.1002/bjs.1800841211

10. Purkayastha S, Tilney HS, Georgiou P, Athanasiou T, Tekkis PP, Darzi AW Laparoscopic cholecystectomy versus minilaparotomy cholecystectomy: a meta-analysis of randomized control trials. Surg Endosc. 2007;21(8):1294-300. https://doi. org/10.1007/s00464-007-9210-3

11. Vagenas K, Spyrakopoulos P, Karanikolas M, Sakelaropoulos G, Maroulis I, Karavias D. Mini-laparotomy cholecystectomy versus laparoscopic cholecystectomy: which way to go? Surg Laparosc Endosc Percutan Tech. 2006;16(5):321-4. https://doi. org/10.1097/01.sle.0000213720.42215.7b

12. Barkun JS, Barkun AN, Sampalis JS, et al. Randomised controlled trial of laparoscopic versus mini cholecystectomy. Lancet. 1992;340(8828):1116-9. https://doi.org/10.1016/01406736(92)93148-g

13. Kiviluoto T, Sire'n J, Luukkonen P, et al. Randomised trial of laparoscopic versus open cholecystectomy for acute and gangrenous cholecystitis. Lancet. 1998;351(9099):321-5. https://doi.org/10.1016/s0140-6736(97)08447-x

14. Shulutko AM, Kazaryan AM, Aghadzanov VG. Minilaparotomy cholecystectomy: Technique, outcomes: A prospective study. Int J Surg. 2007;5(6):423-8. https:// doi:10.1016/j.ijsu.2007.07.004

15. Mbatha SZ, Anderson F. Outcomes in laparoscopic 
cholecystectomy in resource constrained environment. S Afr J Surg. 2016 (accessed on: 21 Dec 2016);54(2):8-12.

16. Shapiro AJ, Costello C, Harkabus M, North JH. Predicting conversion of Laparoscopic Cholecystectomy for acute cholecystitis. JSLS. 1999;3(2):127-30.

17. Botaitis S, Polychronidis A, Pitiakoudis M, Perente S, Simopoulos C. Does gender affect laparoscopic cholecystectomy? Surg Laparosc Endosc Percutan Tech. 2008;18(2):157-61. https://doi. org/10.1097/sle.0b013e318165c899

18. Thesbjerg SE, Harboe KM, Bardram L, Rosenberg J. Sex differences in laparoscopic cholecystectomy. Surg Endosc. 2010;24(12):3068-72. https://doi.org/10.1007/s00464-0101091-1

19. Amber PC, Weber SA, Wassenberg D. Is gallbladder inflammation more severe in male patients presenting with acute cholecystitis. BMC Surg. 2015;15(48). https://doi: 10.1186/ s12893-015-0034-0

20. Syrakos, T., Antonitsis, P., Zacharakis, E. et al. Small-incision (mini-laparotomy) versus laparoscopic cholecystectomy: a retrospective study in a university hospital. Langenbecks. Arch Surg. 2004;389(3):172-7. https://doi:10.1007/s00423-004$0481-\mathrm{z}$

21. Wu YV, Linehan DC. Bile duct injuries in the era of laparoscopic cholecystectomies. Surg Clin N Am. 2010;90(4):787-802. https://doi.org/10.1016/j.suc.2010.04.019

22. Keus F, de Jonge T, Gooszen HG, Buskens E, Van Laarhoven CJ. Cost-minimization analysis in a blind randomized trial on small-incision versus laparoscopic cholecystectomy from a societal perspective: sick leave outweighs efforts in hospital savings. Trials. 2009;10(80). https://doi:10.1186/1745-6215-1080

23. Calvert NW, Troy GP, Johnson AG. Laparoscopic cholecystectomy: a good buy? A cost comparison with small incision (mini) cholecystectomy. Eur J Surg. 2000;166(10):782-6. https://doi: 10.1080/110241500447416 